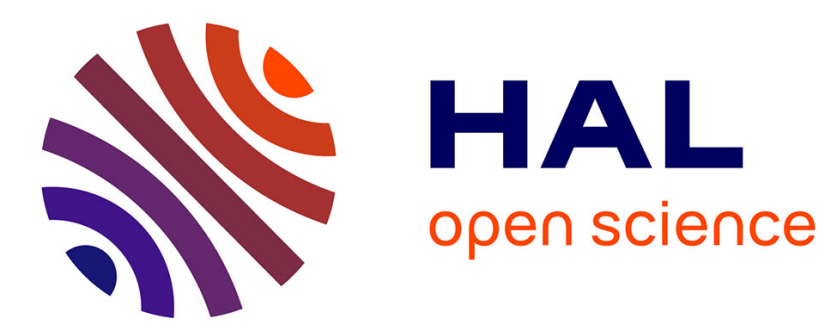

\title{
Normal state resistivity and superconductivity in Pd1- yNiyDx alloys
}

L. Sniadower, L. Dumoulin, P. Nedellec, J.P. Burger

\section{To cite this version:}

L. Sniadower, L. Dumoulin, P. Nedellec, J.P. Burger. Normal state resistivity and superconductivity in Pd1- yNiyDx alloys. Journal de Physique Lettres, 1981, 42 (1), pp.13-16. 10.1051/jphyslet:0198100420101300 . jpa-00231860

\section{HAL Id: jpa-00231860 https://hal.science/jpa-00231860}

Submitted on 1 Jan 1981

HAL is a multi-disciplinary open access archive for the deposit and dissemination of scientific research documents, whether they are published or not. The documents may come from teaching and research institutions in France or abroad, or from public or private research centers.
L'archive ouverte pluridisciplinaire HAL, est destinée au dépôt et à la diffusion de documents scientifiques de niveau recherche, publiés ou non, émanant des établissements d'enseignement et de recherche français ou étrangers, des laboratoires publics ou privés. 


\title{
Normal state resistivity and superconductivity in $\mathrm{Pd}_{1-y} \mathrm{Ni}_{y} \mathrm{D}_{x}$ alloys
}

\author{
L. Sniadower (*), L. Dumoulin, P. Nedellec and J. P. Burger \\ Laboratoire de Physique des Solides, Université Paris-Sud, 91405 Orsay, France
}

(Reçule 11 septembre 1980, accepté le 14 novembre 1980)

\begin{abstract}
Résumé. - Nous mesurons la contribution des phonons optiques à la résistivité électrique d'alliages $\operatorname{Pd}_{1-y} \mathrm{Ni}_{y} \mathrm{D}_{x}$. Cette contribution diminue avec la concentration en nickel en même temps que la température de transition supraconductrice $T_{\mathrm{c}}$. Une analyse quantitative montre que la décroissance de $T_{\mathrm{c}}$ ne peut être entièrement expliquée par la diminution de la contribution des phonons optiques en couplage electron-phonon. D'autres possibilités sont discutées.
\end{abstract}

\begin{abstract}
The contribution of optical phonon scattering to the electrical resistivity of $\mathrm{Pd}_{1-y} \mathrm{Ni}_{y} \mathrm{D}_{x}$ alloys is determined. This contribution decreases with increasing Ni concentration and is accompanied by a depression of the superconducting transition temperature $T_{\mathrm{c}}$. A quantitative analysis of our data indicates that the depression of $T_{\mathrm{c}}$ cannot be entirely accounted for by variation of the contribution of the optical phonons to the electronphonon coupling. Other possible mechanisms are discussed.
\end{abstract}

1. Introduction. - The discovery of superconductivity in $\mathrm{PdH}_{x}$ systems [1, 2] raised the question of the origin of the superconducting state in a material with a low electronic density of states. The positive isotope effect observed for the transition temperature $T_{\mathrm{c}}$ [2], tunnelling experiments $[3,4]$ and normal state resistivity measurements $[5,6]$ demonstrated the importance of the coupling between conduction electrons and optical phonons or $\mathbf{H}$ vibrations. Furthermore, resistivity data indicate that the optical phonon contribution to the electron-phonon coupling parameter is 2 to 4 times larger than that arising from acoustical phonons. This result, which is supported by theoretical considerations [7], indicates that the main factors governing $T_{\mathrm{c}}$ are the density of electron states at the $\mathrm{H}$ site and the value of the optical phonon energy, which is much lower in $\mathrm{PdH}_{x}$ than in most other hydrides.

The first attempts to see how $T_{\mathrm{c}}$ is changed by substitution of Pd with other elements were made by Stritzker [8]. They indicated a substantial increase of $T_{\mathrm{c}}$ for the monovalent elements $\mathrm{Cu}, \mathrm{Ag}$ and $\mathrm{Au}$. One possible explanation is an increase in the $\mathrm{H}$ site density of states. Amongst the elements which can substitute $\mathrm{Pd}$ are the isoelectronic elements, $\mathrm{Ni}$ and

$\left(^{*}\right)$ On leave from Institute of Physics, Polish Academy of Sciences, Warsaw, Poland.
Pt [9]. Whilst no change in $T_{\mathrm{c}}$ is observed for low Pt concentration, $T_{c}$ decreases strongly for $\mathrm{Ni}$ and extrapolates to zero for about $14 \%$ of $\mathrm{Ni}$. This decrease of $T_{\mathrm{c}}$ should be compared with that observed for magnetic impurities, such as $\mathrm{Fe}$ or $\mathrm{Cr}$ for which $T_{\mathrm{c}}$ extrapolates to zero for concentrations below $0.1 \%[10]$.

These data raise the question, what causes the drop, in $T_{\mathrm{c}}$ with increased $\mathrm{Ni}$ concentration. Is it due to a decrease of the optical phonon contribution to $\lambda$ which in turn can be related either to electronic effects (a decrease in the $\mathrm{H}$ site density of states) or to a hardening of the optical phonons ? Are their magnetic effects caused by the high concentration of $\mathrm{Ni}$ despite the fact that it is a non magnetic impurity in most of the simple host metals ?

To answer these questions we present some data on $\mathrm{Pd}_{1-y} \mathrm{Ni}_{y} \mathrm{D}_{x}$ alloys where we measure simultaneously the value of $T_{\mathrm{c}}$ and the optical phonon contribution to the electrical resistivity and so to $\lambda$. The resistivity technique, contrary to tunnelling density of states measurements, can be applied to non superconducting materials, and, indeed, is used here for the sample of highest Ni concentration, which is non superconducting.

2. Experiment. - The Pd-Ni films are prepared on a glass substrate by co-evaporation of $\mathrm{Pd}$ and $\mathrm{Ni}$ using two electron guns in high vacuum $\left(10^{-8}\right.$ torr). 
A nominal composition, $y$, is obtained by monitoring the evaporation rates of both elements using a quartz monitor. We have made two measurements to check this composition. First, we determine the ferromagnetic transition temperature $T_{\mathrm{cm}}$ from the variation with temperature of the extraordinary Hall coeffcient. This gives us an estimate of $y$ independent of the quartz monitor through the well-established $T_{\mathrm{cm}}(y)$ relation [11-12]. Second, we analyse our films by means of the Rutherford backscattering technique. This gives us another estimate of $y$ and in addition, it shows that the homogeneity of our alloys is better then $5 \%$ of $y$. Table I summarizes our data for the two alloys studied.

Table I. - The composition, y, of samples as determined by several different methods, specified in the table. $T_{\mathrm{cm}}$ is the magnetic transition temperature.

\begin{tabular}{cccc}
$\begin{array}{c}\text { Quartz monitor } \\
\text { (nominal } y \text { ) }\end{array}$ & \multicolumn{2}{c}{$\begin{array}{c}\text { Extraordinary Hall } \\
\text { coefficient }\end{array}$} \\
- & $T_{\text {cm }}(\mathrm{K})$ & $y$ & $\begin{array}{c}\text { Rutherford } \\
\text { backscattering }\end{array}$ \\
0.09 & - & - & - \\
0.25 & 143 & $0.089 \pm 0.002$ & $0.086 \pm 0.003$ \\
& 303 & $0.24 \pm 0.01$ & $0.23 \pm 0.01$
\end{tabular}

The deposited films were electrolitically hydrogenated at a temperature of about $-80^{\circ} \mathrm{C}$, directly inside the helium cryostat as described elsewhere $[4,13]$. The charging procedure is controlled by monitoring the film resistance as a function of charging time. This resistance goes through a maximum and then drops to a final value which for the most Ni rich alloys $(y=8$ and $25 \%)$ is lower then the initial resistance i.e. that for $x=0$. We have not measured the deuterium concentration, $x$, but by considering the residual resistivity due to deuterium vacancies, and the residual resistivity of $\mathrm{Ni}$ in $\mathrm{Pd}$ and in $\mathrm{PdD}_{x}$, we deduce that $x$ is almost the same for all alloys and is close to $x=1$. The exact value of $x$ is not important in these studies, as will become evident in the following sections, but it is because we compare the change of $T_{\mathrm{c}}$ with that of the electronoptical phonon coupling parameter $\lambda_{\text {op }}$ for the same deuterium concentration, $x$.

We prefer to use deuterium instead of hydrogen because there is a correspondingly larger contribution from optical phonon scattering to the resistivity in the temperature range, $50 \mathrm{~K}<T<100 \mathrm{~K}$, for which there is no loss of $\mathrm{D}$ or $\mathrm{H}$ from our films, a few hundreds $\AA$ thick, ( $T_{\max }$ on figure 1 represents the maximum temperature of our resistivity measurements).

3. Analysis of the experimental data. - The phonon electrical resistivity $\rho(T)$ and the superconducting transition temperature, $T_{\mathrm{c}}$, can be written in the following way :

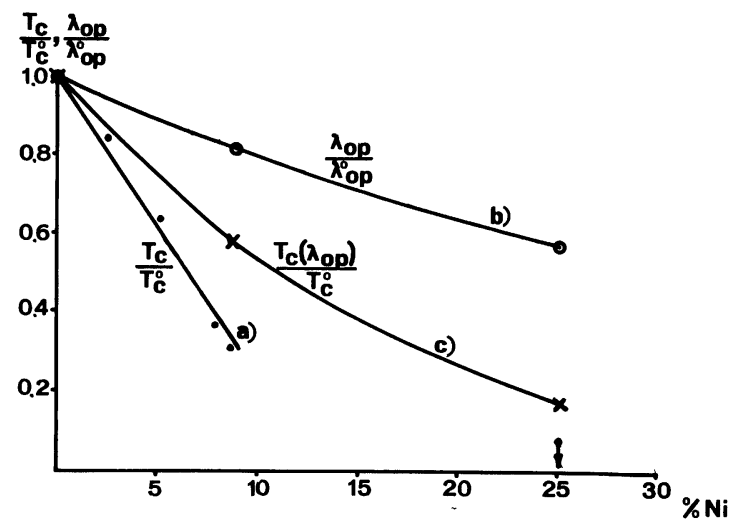

Fig. 1. - Experimental superconducting transition temperature $T_{\mathrm{c}}(1 a)$, electron-optical phonon coupling parameter $\lambda_{\mathrm{op}}(1 b)$ and $T_{\mathrm{c}}\left(\lambda_{\mathrm{op}}\right)$ calculated from $\lambda_{\mathrm{op}}$ using the Mc Millan formula $(1 c)$, as a function of $\mathrm{Ni}$ concentration. All values are normalized to the corresponding value for $y=0$.

$$
\begin{gathered}
\rho(T)=C \int \alpha_{\mathrm{t}}^{2} F(\omega) f\left(\frac{\hbar \omega}{k T}\right) \mathrm{d} \omega, \\
T_{\mathrm{c}}=\langle\omega\rangle \exp \left(-\frac{1}{g}\right), \quad g=\frac{\lambda}{1+\lambda}-\mu^{*}, \\
\lambda=2 \int \frac{\alpha^{2} F(\omega)}{\omega} \mathrm{d} \omega .
\end{gathered}
$$

$C$ is a constant involving the effective mass and the number density of conduction electrons, $F(\omega)$ is the phonon density of states, $\alpha_{t}^{2}$ is a transport electronphonon coupling matrix element which differs from the $\alpha^{2}$ appearing in $\lambda$ by a factor involving the scattering angle, and

$$
f(x)=\frac{x}{\left(\mathrm{e}^{x}-1\right)\left(1-\mathrm{e}^{-x}\right)}
$$

is a statistical factor. The expression for $T_{\mathrm{c}}$ is the standard McMillan type formula.

At high enough temperatures $f(x) \simeq 1 / x$ and $\rho(T) \simeq A T$ where $A$ is proportional to

$$
\lambda_{\mathrm{t}}=2 \int \frac{\alpha_{\mathrm{t}}^{2} F(\omega)}{\omega} \mathrm{d} \omega \simeq \lambda .
$$

Thus, it is possible to extract $\lambda$ from the measured values of $\rho(T)$. However, for compounds like $\mathrm{Pd}_{1-y} \mathrm{Ni}_{y} \mathrm{D}_{x}$ we have to consider contributions to $\rho(T)$ both from acoustic and optic phonons i.e.

and

$$
\begin{gathered}
\rho(T)=\rho_{\mathrm{ac}}(T)+\rho_{\mathrm{op}}(T) \\
\lambda=\lambda_{\mathrm{ac}}+\lambda_{\mathrm{op}} .
\end{gathered}
$$

It is possible to separate the two contributions because at low enough temperatures $(T<50 \mathrm{~K})$ only acoustical phonons are thermally excited, so that $\rho(T)=\rho_{\mathrm{ac}}(T)$. Approximating $\rho(T)$ in this temperature range by a Grüneisen function,

$$
\rho_{\mathrm{ac}}(T)=\rho_{\mathrm{G}}\left(T / \theta_{\mathrm{ac}}, A_{\mathrm{ac}}\right),
$$


and assuming that this approximation is also valid at higher temperatures, it is possible to isolate the optical phonon contribution over the whole temperature interval considered i.e.

$$
\rho_{\mathrm{op}}(T)=\rho(T)-\rho_{\mathrm{ac}}(T) .
$$

The optical contribution is then analysed by approximating the optical phonon spectrum with an Einstein spectrum i.e. $\rho_{\mathrm{op}}(T)=\rho_{\mathrm{E}}\left(T / \theta_{\mathrm{op}}, A_{\mathrm{op}}\right)$. A best fit of $\rho_{\mathrm{ac}}(T)$ and $\rho_{\mathrm{op}}(T)$ with $\rho_{\mathrm{G}}$ and $\rho_{\mathrm{E}}$ permits us to determine $\theta_{\mathrm{ac}}$ (the Debye temperature), $\theta_{\text {op }}$ (the Einstein temperature), $A_{\mathrm{ac}}$ and $A_{\mathrm{op}}$. Since the constant $C$ is not known precisely, one can determine only the ratio $\lambda_{\mathrm{op}} / \lambda_{\mathrm{ac}}=A_{\mathrm{op}} / A_{\mathrm{ac}}$. Absolute values of $\lambda_{\mathrm{op}}$ and $\lambda_{\mathrm{ac}}$ can nevertheless be obtained by calculating the corresponding values $\lambda_{\text {op }}^{0}$ and $\lambda_{\text {ac }}^{0}$ for the nickel free compound using relations (2) and (3), for which we assume $\mu^{*}=0.1$, a typical value of the electronelectron repulsive interaction parameter in non transition metals and $\langle\omega\rangle=\left(\theta_{\mathrm{ac}}\right)^{\lambda_{\mathrm{ac}} / \lambda}\left(\theta_{\mathrm{op}}\right)^{\lambda_{\mathrm{op}} / \lambda}$.

4. Results and discussion. - Figure $1 a$ shows the decrease of $T_{\mathrm{c}}$ with $y$ for several deuterated samples including those two for which we have also measured the resistivities. These data are in good agreement with results obtained by Stritzker [9] and indicate that $T_{\mathrm{c}}$ approaches zero for a nickel concentration close to $13 \%$. No superconductivity has been observed in the $y=25 \%$ sample down to $1.1 \mathrm{~K}$ : this is again in agreement with the data of Stritzker but not with those of Skośkiéwicz $[14,9]$. We have no explanation for this discrepancy. Following the procedure outlined above, we show in figure 2 the contribution $\rho_{\text {op }}(T)$ arising from optical phonon scattering for different $\mathrm{Ni}$ concentrations $y=(0,0.09,0.25)$. The procedure for separating the optical and acoustical phonon contributions is illustrated in this figure for the $y=0$ case. At first sight one observes a decrease of $\rho_{\text {op }}(T)$ with $y$. The numerical analysis shows that one can obtain a good fit for $\rho_{\text {op }}(T)$ using an Einstein spectrum. This fit indicates that the decrease of $\rho_{\text {op }}(T)$ cannot be attributed to a hardening of the optical phonons. If the electron-phonon coupling parameter is written in the usual form

$$
\lambda_{\mathrm{op}}=\frac{\eta_{\mathrm{op}}}{M \omega_{\mathrm{op}}^{2}}
$$

our data imply instead a decrease of $\eta_{\text {op }}$ with $y$. The decrease of $\lambda_{\mathrm{op}}$ (normalized to $\lambda_{\mathrm{op}}^{0}$ when $y=0$ ) is shown in figure $1 b$ (we have not drawn $\lambda_{\mathrm{ac}}(y)$, which depends only slightly on $y$ ).

One can try to relate the change of $\lambda_{\text {op }}$ with $y$ with changes in the electronic band structure accompanying the replacement of Pd by Ni. Recent theoretical band structure calculations [15] made on pure $\mathrm{PdH}$ and $\mathrm{NiH}$ compounds show that $\eta_{\mathrm{op}}$ is sensitive primarily to the $\mathrm{H}$ site Fermi level density of states, and an important difference between $\mathrm{PdH}$ and $\mathrm{NiH}$ is that

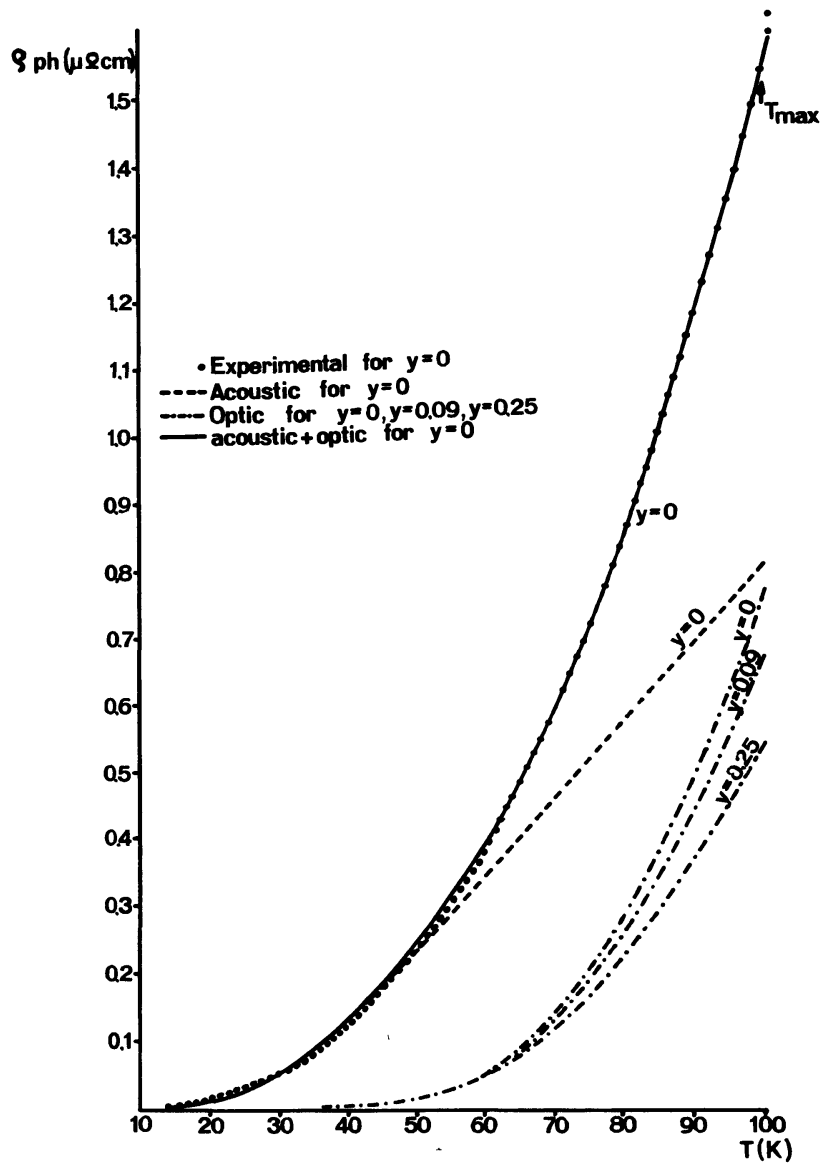

Fig. 2. - Contributions to phonon resistivity arising from optical phonon scattering $(\cdot-\cdot-\cdot-)$ for different $\mathrm{Ni}$ concentrations $y$. To illustrate how the separation procedure described in the text works, the complete data for $y=0$ are also shown. The acoustical contribution $(---)$ is a theoretical Grüneisen law fitted to the low temperature resistivity. The optical contribution is obtained by subtracting the acoustical contribution from the experimental resistivity. The solid line represents the best fit to the data of the sum of the theoretical acoustic + optical contributions (see text).

the Fermi level is much closer to the top of the $d$ band in $\mathrm{NiH}$ which will result in a lower type density of states at the $\mathrm{H}$ site for $\mathrm{NiH}$ compared to $\mathrm{PdH}$. The calculated value of $\eta_{\mathrm{op}}$ is more than two times smaller for $\mathrm{NiH}$ and thus could possibly account for the observed decrease of $\lambda_{\text {op }}$ with $y$.

However it is not certain that such a decrease in $\lambda_{\text {op }}$ would lead to the observed large decrease of $T_{\mathrm{c}}$. If one compares, for instance, the present data with those observed on $\operatorname{PdD}_{x}$ alloys [13] where there is a similar correlation between $T_{\mathrm{c}}(x)$ and $\lambda_{\mathrm{op}}(x)$, one finds that

$$
\left|\frac{\mathrm{d} \lambda_{\mathrm{op}}(y)}{\mathrm{d} T_{\mathrm{c}}}\right|<\left|\frac{\mathrm{d} \lambda_{\mathrm{op}}(x)}{\mathrm{d} T_{\mathrm{c}}}\right|,
$$

which means that the change of $\lambda_{\text {op }}$ for a given change of $T_{\mathrm{c}}$ is smaller as a function of the nickel concentration $y$ than as a function of the deuterium concentration $x$. To quantify this observation we calculate by how much $T_{\mathrm{c}}$ should decrease with $y$ using the mea- 
sured changes in $\lambda_{\text {op }}$ (Fig. 1b). The calculated values of $T_{\mathrm{c}}$ are reported in figure $1 c$, and lie above the observed $T_{\mathrm{c}}$ points which suggests that part of the variation of $T_{\mathrm{c}}(y)$ results from some other cause. First consider the acoustical contribution $\lambda_{\text {ac }}$; experiments show that this term is small while theory [15] indicates that $\eta_{\mathrm{ac}}$ is similar in both $\mathrm{PdH}$ and $\mathrm{NiH}$. Therefore this term is probably not relevant. A second possibility is some sort of magnetic contribution to the change of $T_{\mathrm{c}}$. Ni is not magnetic in $\mathrm{PdH}$ in the sense that there is no local moment on the Ni atoms. However, it has been shown that transition metal impurities which do not form local moments produce nevertheless a depression of $T_{\mathrm{c}}$, which is smaller than that for magnetic impurities, and which is related to the Coulomb repulsion between localized d electrons of opposite spins [16] or, possibly to localized magnetic fluctuations [16]. The calculated value of $\Delta T_{\mathrm{c}} / T_{\mathrm{c}}$ is expected to be of the order of 0.1 per percent of $\mathrm{Ni}$, a value large enough to explain the observed decrease of $T_{\mathrm{c}}$ [16]. Note also that such a decrease of $T_{\mathrm{c}}$ is not observed for Pt which is isoelectronic to $\mathrm{Ni}$ but which shows a smaller tendency towards formation of magnetic moments than Ni. One can also argue that the tendency towards magnetism which can be described by the value of the Kondo temperature, $T_{\mathrm{K}}$, is more pronounced for the matrix $\mathrm{PdH}$ than for a classical monovalent matrix. For instance, Co impurities are non magnetic in $\mathrm{Cu}$ (very high $T_{\mathrm{K}}$ ) whereas they are more magnetic in $\mathrm{PdH}_{x}\left(T_{\mathbf{K}}\right.$ in the range of 1 to $\left.10 \mathrm{~K}\right)$ [17]. Finally note that a decrease of $T_{\mathrm{c}}$ of the same order of magnitude as in $\mathrm{Pd}_{1-y} \mathrm{Ni}_{y} \mathrm{D}_{x}$ alloys is also seen in the $\mathrm{Al}-\mathrm{Ni}$ alloys $[16,18]$ despite the fact that $\mathrm{d}$ transition metal impurities form no magnetic moment in the $\mathrm{Al}$ matrix.

In conclusion we observe a large decrease in $T_{\mathrm{c}}$ with increasing nickel concentration in $\operatorname{Pd}_{1-y} \mathrm{Ni}_{y} \mathrm{D}_{x}$ alloys. Only a part of this variation can be attributed to a decrease of the coupling of electrons to the optical phonons and the total variation in $T_{\mathrm{c}}(y)$ can only be explained if one includes some other effect related, most probably, to the magnetic structure of the $\mathrm{Ni}$ impurity. Tunnelling measurements on these same alloys are in progress to try to explain this problem.

Acknowledgments. - We would like to thank C. Cohen and F. Zawislak for performing and analysing the backscattering experiments and H. Bernas, J. Chaumont and A. J. Pindor for useful discussions.

\section{References}

[1] Skośkiéwicz, T., Phys. Status Solidi (a) 11 (1972) K 123.

[2] Stritzker, B., Buckel, W., Z. Phys. 257 (1972) 1.

[3] Eichler, A., WüHL, H., Stritzker, B., Solid State Commun. 17 (1975) 213.

[4] Dumoulin, L., Nedellec, P., Arzoumanian, C., Burger, J. P., Phys. Status Solidi (b) 90 (1978) 207.

[5] Mac Lachlan, D. S., Mailfert, R., Burger, J. P., SoufFACHE, B., Solid State Commun. 17 (1975) 281.

[6] Górska, A., Górski, A. M., Igalson, J., Pindor, A. J., Sniadower, L., Proc. of 2nd Int. Congress "Hydrogen in Metals", Paris, 2A10 (1977).

[7] Papaconstantopoulos, D. A., Klein, B. M., Phys. Rev. Lett. 35 (1975) 110 ;

Papaconstantopoulos, D. A., Klein, B. M., Economou E. N., BoYer, L. L., Phys. Rev. B 17 (1978) 141.

[8] Stritzker, B., Z. Phys. 268 (1974) 261.

[9] StritzKer, B., WüHL, H., in " Hydrogen in Metals », Topics in Applied Physics, Vol. 29 II (Springer Verlag) 1978, p. 243.

[10] Van Dongen, T. M., Mydosh, T. A., Z. Phys. Chem. Bd 116 (1979) 149.

[11] LANDolT-BöRnstein, Springer Verlag II band 9 Teil : Magnetische Eigenscheften I (1962) 1-56.

[12] Crangle, J., Scott, W. R., J. Appl. Phys. 36 (1965) 921.

[13] Arzoumanian, C., Burger, J. P., Dumoulin, L., NedelLEC, P., Z. Phys. Chem. 116 (1979) 117.

[14] Skośkiéwicz, T., Proc. 5th Intern. Conf. High Pressure and Technology, Moscow, 1975.

[15] Gupta, M., Burger, J. P., J. Phys. F, in press.

[16] KAISER, A. B., thesis work, University of London (1971).

[17] Senoussi, S., Burger, J. P., Souffache, B., Phys. Lett. 54A (1975) 288.

[18] Aoki, R., Ohtsuka, T., J. Phys. Soc. Japan. 23 (1967) 955 ; 26 (1969) 651. 